\title{
The wandering spleen torsion
}

\author{
Irpatgire Ravindra $\mathbf{N}^{1}$, Kale $\mathbf{D}^{2}$
}

${ }^{1}$ Dr Irpatgire Ravindra N, Associate Professor, MIMSR Medical College, Latur, Maharashtra, India, ${ }^{2}$ Dr Dinkar Kale, Professor, MIMSR Medical College, Latur, Maharashtra, India

Address for Correspondence: Dr Irpatgire Ravindra N, Associate Professor, MIMSR Medical College Latur, Maharashtra, India, Email id: rirpatgire@ @ rediffmail.com.

\begin{abstract}
Wandering spleen is the absence of spleen at its normal left upper quadrant position. It may be because of increased splenic mobility resulting from laxity or maldevelopment of the suspensary gastrosplenic, splenorenal, and phrenicocolic ligaments. Wandering spleen is prone for complications like trauma and torsion. Torsion is quite rare complication. Diagnosis of wandering spleen is quite difficult because of the lack of symptoms and signs until splenic torsion have occurred. Here we report an interesting case of a 16-year-old unmarried girl presenting with acute abdomen, diagnosed with wandering spleen torsion and managed surgically.
\end{abstract}

Keywords: Wandering Spleen, Ectopic Spleen, Torsion, Twisted Pedicle.

\section{Introduction}

Wandering spleen is the absence of the spleen in its normal left upper quadrant position. It may be secondary to the congenital or functional absence of the splenic ligament. Torsion of the wandering spleen is rare with fewer than 500 cases reported and incidence of less than $0.2 \%$ [1]. It is common in 20 to 40 years of age and majority cases are women [2]. We reported a case of 16 year old unmarried girl presented with acute pain in abdomen and features of hypersplenism. Finally she has been diagnosed to have wandering spleen with its torsion and infraction.

\section{Case Report}

A 16 year girl presented with complain of pain in abdomen since ten days. Sudden onset pain was continuous and more in the left lumbar and iliac region. There was no history of vomiting, bowel or urinary symptoms. She was febrile and her vital signs were stable. Abdominal examination revealed a large

Manuscript received: $16^{\text {th }}$ Oct 2015

Reviewed: $25^{\text {th }}$ Oct 2015

Author Corrected: $11^{\text {th }}$ Nov 2015

Accepted for Publication: $28^{\text {th }}$ Nov 2015 abdominal tender mass in left lumbar region. Urinalysis and urine culture were normal. Her WBC count was 16 $\times 10^{9} / \mathrm{L}$, haemoglobin $5.0 \mathrm{~g} / \mathrm{dL}$ and Platelets $150 \times$ $10^{9} / \mathrm{L}$. On ultrasound, spleen was absent at its normal position in left upper quadrant. Spleen was found below the lower pole of the left kidney. A large hypoechoic area was seen, suggestive of infarction, with minimal perisplenic fluid collection. Colour Doppler demonstrated no blood flow in the splenic vein and artery indicating spleen is not viable. Subsequent contrast-enhanced CT scan was done to confirm the ultrasound findings. It also showed the presence of the spleen below lower pole of the left kidney with torsion of spleen. [Fig. 1\&2]. Contrast enhanced CT shows infarction and confirmed that spleen is not viable. The patient underwent exploratory laparotomy through a midline incision. This revealed the absence of spleen at its normal position. Bigger and congested spleen found in the left iliac region below lower pole of the left kidney. All splenic ligaments and short gastric vessels were absent. Splenic pedicle was found to be unduly long [Fig 3] and twisted at the hilum leading to infraction and nonviable spleen. Total splenectomy was performed in view of non viability of the spleen. The patient's post-operative course was uneventful. 


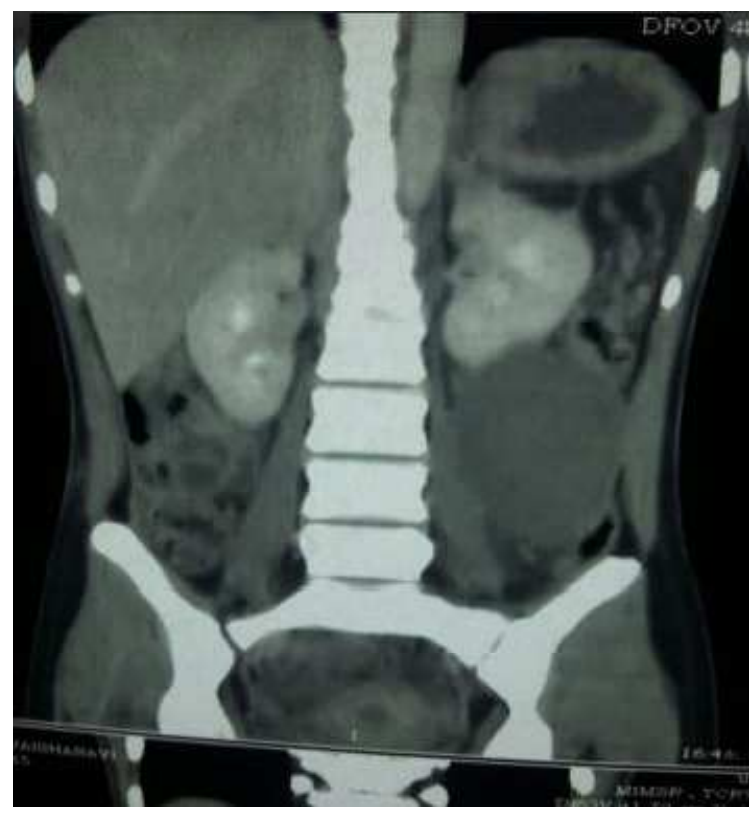

Figure 1: Spleen below lower pole of the left kidney

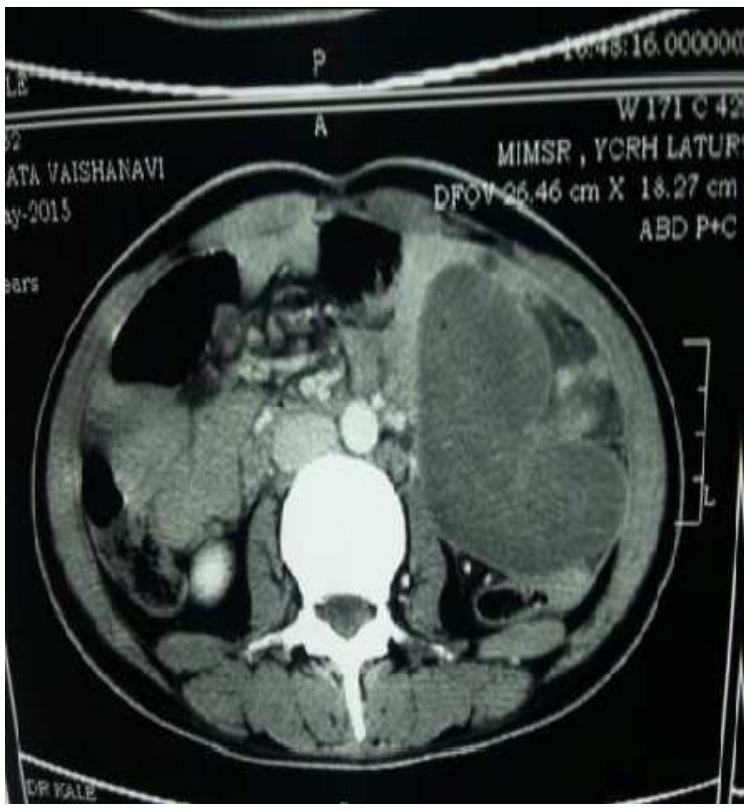

Figure 2: Splenic torsion with hilum facing anterolateral

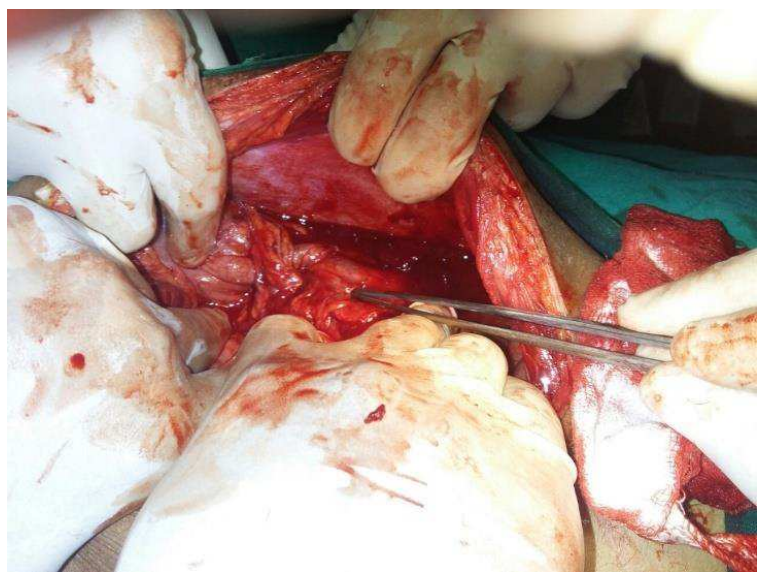

Figure 3: long twisted splenic vascular pedicle

\section{Discussion}

Wandering spleen is rare condition. Van home described a case of wandering spleen for the first time in 1667 while performing an autopsy. It is common between age 20 to 40 years and $70 \%$ to $80 \%$ cases are seen in women in childbearing age $[2,3]$.

Wandering spleen may be either congenital or acquired. Congenital wandering spleen is attributed to failure of fusion of the dorsal mesogastrium during the 5th and 6th week of development resulting in an unusually long splenic pedicle. Suspensary gastrosplenic, splenorenal, and phrenicocolic ligaments are main supports of spleen. Absence of ligaments or failure of these fusions leads to excessive mobility resulting into congenital wandering spleen. The long splenic pedicle increases hypermobility of spleen thus increases chance for the torsion [4]. Wandering spleen has also been seen in prun belly syndrome.[5]. The acquired form occurs in multiparous women possibly as result of hormonal changes in pregnancy that causes laxity of the abdominal wall and ligaments attached to spleen.[3,6] Some case reports have included a history of malaria, trauma and hematological disease as associated etiology [7].

Diagnosis of wandering spleen is difficult due its rare occurrence and nonspecific symptoms [2,3]. Majority of the patients present with asymptomatic mass. They may also present with nonspecific GIT complaints. Rarely few patients present with an acute abdomen secondary to splenic torsion and infraction [2]. Mechanical factors resulting in urinary retention, Intestinal obstruction or symptoms due to pathological disturbances of Spleen 
such as thrombocytopenia, hypersplenism have been described [1]. Other sporadic presentation described are gastric volvulus, variceal hemorrhage and rarely acute pancreatitis [8]. Splenic torsion may be acute on chronic. Acute torsion presents as classic acute abdomen and mimic peritonitis, acute appendicitis, twisted ovarian cyst, diverticulitis, cholecystitis and intestinal obstruction.

Since clinical diagnosis may be difficult, a definitive diagnosis of wandering spleen depends on imaging like USG, CT Scan and MRI. Ultrasound and the scintigraphy are the initial imaging studies of choice with scintigraphy being useful to demonstrate splenic function. If torsion is suspected, CT with contrast can diagnose infarction represented by splenic attenuation lower than that of the liver. A classic "whorl sign" on CT is characteristic of splenic torsion. It is described as "the presence of a twisted splenic pedicle intermingled with fat resulting in alternate circular bands of radio density and radiolucency [9].

The treatment of choice for wandering spleen is splenopexy where diagnosis is made early before infarction and necrosis have occurred [10]. Formerly splenectomy was the treatment of choice; however, concerns over the post splenectomy sepsis have elevated splenopexy to the desired first line treatment. Splenectomy is done if there is functional asplenia due to torsion, splenic infraction, splenic vessel thrombosis, hypersplenism, as in our case. It is also advised where huge splenomegaly precluding splenopexy or any suspicion of malignancy [2].

\section{Conclusion}

A high index suspicion through clinical examination combined with ultrasound and CT increases the chance of preoperative diagnosis of wandering spleen. Splenopexy is the treatment of choice when diagnosis made before infarction and necrosis.
Funding: None, Competing interests: None declared Ethical clearance: The study was approved by the institutional human ethical committee.

\section{References}

1. Satyadas T, Nasir N, Bradpiece HA. Wandering spleen: case report and literature review. J R Coll Surg Edinb. 2002 Apr;47(2):512-4.

2. Buehner M, Baker MS. The wandering spleen. Surg Gynecol Obstet. 1992 Oct;175(4):373-87.

3. Desai DC, Hebra A, Davidoff AM, Schnaufer L. Wandering spleen: a challenging diagnosis. South Med J. 1997 Apr;90(4):439-43.

4. Lewis GA, Byrne MP. Wandering spleen. Am Surg. 1981 Jun;47(6):275-7.

5. Aliabad H, Foker J, Gonzalez R. Splenic torsion and the prun belly syndrome. Ped Surg Int 1987; 2:369-71.

6. Carswell JW. Wandering spleen: 11 cases from Uganda. Br J Surg. 1974 Jun;61(6):495-7.

7. Abell I. Wandering spleen with torsion of the pedicle. Ann Surg. 1933 Oct;98(4):722-35.

8. Daneshgar S, Eras P, Feldman SM, Cacace VA, Federico FN, Levin RH. Bleeding gastric varices and gastric torsion secondary to a wandering spleen. Gastroenterology. 1980 Jul;79(1):141-3.

9. Thambidorai CR, Imtiaz A, Nafiqudin M. Torsion of a wandering spleen with whorled appearance of the splenic hilum in CT scan. Med J Malaysia. 2005 Dec;60(5):653-4.

10. McHeik JN, Richer JP, Levard G. [Torsion of the spleen in children]. Arch Pediatr. 2005 Oct;12(10):1496-9. Epub

\section{How to cite this article?}

Irpatgire Ravindra N, Kale D. The wandering spleen torsion. Int J Med Res Rev 2015;3(10):1276-1278. doi: 10.17511/ijmrr.2015.i10.232. 